UDC 517.944

\title{
GENERALIZED EULER METHOD FOR NONLINEAR FIRST ORDER PARTIAL DIFFERENTIAL EQUATIONS
}

УЗАГАЛЬНЕНИЙ МЕТОД ЕЙЛЕРА ДЛЯ НЕЛІНІЙНИХ ДИФЕРЕНЦАЛЬНИХ РІВНЯНЬ З ЧАСТИННИМИ ПОХІДНИМИ ПЕРІІОГО ПОРЯДКУ

\author{
Z. Kamont, J. Newlin-Łukowicz \\ Techn. Univ. Gdańsk \\ 11/12 Gabriel Narutowicz Street, \\ 80-952 Gdańsk, Poland
}

Classical solutions of nonlinear first order partial differential equations are approximated in the paper by solutions of quasilinear systems of difference equations. Sufficient conditions for the convergence of the method are given. The proof of the stability of the difference problem is based on a comparison method. Nonlinear estimates of the Perron type are assumed for increment functions.

This new approach to a numerical solving of nonlinear equations is generated by a method of quasilinearization for mixed problems. Numerical examples are given.

Класичні розв'язки нелінійних диференціальних рівнянь з частинними похідними першого порядку наближаються розв'язками квазілінійних систем різницевих рівнянь. Наведено достатню умову для збіжності запропонованого методу. Доведення стійкості різницевої задачі базуеться на методі порівняння. Вважаеться, щзо функція приросту задовольняе нелінійні оцінки перронівського типу.

Цей новий підхід до числового розв'язання нелінійних рівнянь базується на методі квазілінеаризацї для мішаних задач. Наведено числові приклади.

1. Discretization of mixed problems. For any metric spaces $X$ and $Y$ we denote by $C(X, Y)$ the set of all continuous functions defined on $X$ and taking values in $Y$. We will use vectorial inequalities with the understanding that the same inequalities hold between their corresponding components. Write

$$
E=[0, a] \times[-b, b] \text { and } E_{0}=\{0\} \times[-b, b]
$$

where $a>0, b=\left(b_{1}, \ldots, b_{n}\right) \in R^{n}$ with $b_{i}>0$ for $1 \leq i \leq n$. Set $\Omega=E \times R \times R^{n}$, and suppose that $f: \Omega \rightarrow R$ is a given function of the variables $(t, x, p, q)$ where $x=\left(x_{1}, \ldots, x_{n}\right)$ and $q=\left(q_{1}, \ldots, q_{n}\right)$. Let us assume that $f \in C(\Omega, R)$ and that the first order partial derivatives

$$
\left(\partial_{q_{1}} f(P), \ldots, \partial_{q_{n}} f(P)\right)=\partial_{q} f(P)
$$

exist for $P=(t, x, p, q) \in \Omega$. Write

$$
\partial_{+}^{(i)} E=\left\{(t, x) \in E: x_{i}=b_{i}\right\}, \quad \partial_{-}^{(i)} E=\left\{(t, x) \in E: x_{i}=-b_{i}\right\}, \quad 1 \leq i \leq n,
$$

(c) Z. Kamont, J. Newlin-Łukowicz, 2003 
and

$$
\partial_{0} E=\bigcup_{i=1}^{n}\left(\partial_{+}^{(i)} E \cup \partial_{-}^{(i)} E\right)
$$

In the following we will assume that

$$
\partial_{q_{i}} f(t, x, p, q)>0 \quad \text { for } \quad(t, x, p, q) \in \partial_{+}^{(i)} E \times R \times R^{n}, \quad 1 \leq i \leq n,
$$

and

$$
\partial_{q_{i}} f(t, x, p, q)<0 \quad \text { for } \quad(t, x, p, q) \in \partial_{-}^{(i)} E \times R \times R^{n}, \quad 1 \leq i \leq n .
$$

Suppose that $\varphi: E_{0} \cup \partial_{0} E \rightarrow R$ is a given function. We consider the problem consisting of the nonlinear differential equation

$$
\partial_{t} z(t, x)=f\left(t, x, z(t, x), \partial_{x} z(t, x)\right)
$$

and the initial boundary condition

$$
z(t, x)=\varphi(t, x) \quad \text { for } \quad(t, x) \in E_{0} \cup \partial_{0} E,
$$

where $\partial_{x} z=\left(\partial_{x_{1}} z, \ldots, \partial_{x_{n}} z\right)$. A function $v: E \rightarrow R$ is called a classical solution of the above problem if

(i) $v \in C(E, R)$ and $v$ is of class $C^{1}$ on $E \backslash\left(E_{0} \cup \partial_{0} E\right)$,

(ii) $v$ satisfies (3) on $E \backslash\left(E_{0} \cup \partial_{0} E\right)$ and initial boundary condition (4) holds.

We formulate now a finite difference problem corresponding to (3), (4). Let $\mathbb{N}$ and $\mathbb{Z}$ be the sets of natural numbers and integers, respectively. For $x, y \in R^{n}, x=\left(x_{1}, \ldots, x_{n}\right), y=$ $=\left(y_{1}, \ldots, y_{n}\right)$, we write

$$
x \diamond y=\left(x_{1} y_{1}, \ldots, x_{n} y_{n}\right) \quad \text { and } \quad\|x\|=\sum_{i=1}^{n}\left|x_{i}\right|
$$

We define a mesh on the set $E$ in the following way. Let $\left(h_{0}, h^{\prime}\right), h^{\prime}=\left(h_{1}, \ldots, h_{n}\right)$, stand for steps of the mesh. For $h=\left(h_{0}, h^{\prime}\right)$ and $(r, m) \in \mathbb{Z}^{1+n}$ where $m=\left(m_{1}, \ldots, m_{n}\right)$, we define nodal points as follows:

$$
t^{(r)}=r h_{0}, \quad x^{(m)}=m \diamond h^{\prime}, \quad x^{(m)}=\left(x_{1}^{\left(m_{1}\right)}, \ldots, x_{n}^{\left(m_{n}\right)}\right) .
$$

Let us denote by $\Delta$ the set of all $h=\left(h_{0}, h^{\prime}\right)$ such that there is $N=\left(N_{1}, \ldots, N_{n}\right) \in \mathbb{N}^{n}$ with the property $N \diamond h^{\prime}=b$. We assume that $\Delta \neq \emptyset$. There is $N_{0} \in \mathbb{N}$ such that $N_{0} h_{0} \leq a<\left(N_{0}+1\right) h_{0}$. Write

$$
R_{h}^{1+n}\left\{\left(t^{(r)}, x^{(m)}\right):(r, m) \in \mathbb{Z}^{1+n}\right\}
$$




$$
I_{h}=\left\{t^{(r)}: 0 \leq r \leq N_{0}\right\}
$$

and

$$
\begin{gathered}
E_{h}=E \cap R_{h}^{1+n}, \quad \partial_{0} E_{h}=\partial_{0} E \cap R_{h}^{1+n}, \\
E_{r, h}=E_{h} \cap\left(\left[0, t^{(r)}\right] \times R^{n}\right) \quad \text { where } \quad 0 \leq r \leq N_{0} .
\end{gathered}
$$

For functions $z: E_{h} \rightarrow R, u: E_{h} \rightarrow R^{n}, u=\left(u_{1}, \ldots, u_{n}\right)$, and $\xi: I_{h} \rightarrow R$, we write $z^{(r, m)}=z\left(t^{(r)}, x^{(m)}\right), u^{(r, m)}=u\left(t^{(r)}, x^{(m)}\right), \xi^{(r)}=\xi\left(t^{(r)}\right)$ and

$$
\begin{aligned}
& \|z\|_{r, h}=\max \left\{\left|z^{(i, m)}\right|:\left(t^{(i)}, x^{(m)}\right) \in E_{r, h}\right\}, \\
& \|u\|_{r, h}=\max \left\{\left|u^{(i, m)}\right|:\left(t^{(i)}, x^{(m)}\right) \in E_{r, h}\right\},
\end{aligned}
$$

where $0 \leq r \leq N_{0}$. Let $e_{j}=(0, \ldots, 0,1,0, \ldots, 0) \in R^{n}, 1$ standing in the $j$-th place. We denote by $\delta_{0}$ the difference operator with respect to variable $t$ and by $\delta=\left(\delta_{1}, \ldots, \delta_{n}\right)$ the difference operator for spatial variables $\left(x_{1}, \ldots, x_{n}\right)=x$. Write

$$
\delta_{0} z^{(r, m)}=\frac{1}{h_{0}}\left[z^{(r+1, m)}-\frac{1}{2 n} \sum_{j=1}^{n}\left(z^{\left(r, m+e_{j}\right)}+z^{\left(r, m-e_{j}\right)}\right)\right]
$$

and

$$
\delta_{j} z^{(r, m)}=\frac{1}{2 h_{j}}\left(z^{\left(r, m+e_{j}\right)}-z^{\left(r, m-e_{j}\right)}\right) \quad \text { for } \quad 1 \leq j \leq n .
$$

A classical difference method for the mixed problem consists in replacing partial derivatives in (3) by the above difference operators. This leads to the difference equation

$$
\delta_{0} z^{(r, m)}=f\left(t^{(r)}, x^{(m)}, z^{(r, m)}, \delta z^{(r, m)}\right)
$$

with the initial boundary condition

$$
z^{(r, m)}=\varphi_{h}^{(r, m)} \quad \text { on } \quad E_{0, h} \cup \partial_{0} E_{h}
$$

where $\delta z^{(r, m)}=\left(\delta_{1} z^{(r, m)}, \ldots, \delta_{n} z^{(r, m)}\right)$ and $\varphi_{h}: E_{0, h} \cup \partial_{0} E_{h} \rightarrow R$ is a given function. Sufficient conditions for the convergence of method (7), (8) to a classical solution of (3), (4) are given in the following theorem.

Theorem 1. Suppose that

1) $f \in C(\Omega, R)$, the derivatives $\left(\partial_{q_{1}} f, \ldots, \partial_{q_{n}} f\right)=\partial_{q} f$ exist on $\Omega$ and $\partial_{q} f \in C\left(\Omega, R^{n}\right)$;

2) conditions (1), (2) are satisfied and there is $A \in R_{+}$such that

$$
|f(t, x, p, q)-f(t, x, \bar{p}, q)| \leq A|p-\bar{p}| \quad \text { on } \quad \Omega
$$


3) $h \in \Delta$ and there is $M=\left(M_{1}, \ldots, M_{n}\right) \in R^{n}$ such that $M_{i}>0$ for $1 \leq i \leq n$ and $h^{\prime} \leq M h_{0}$

4) the estimates

$$
1-\frac{n h_{0}}{h_{j}}\left|\partial_{q_{j}} f(t, x, p, q)\right| \geq 0, \quad 1 \leq j \leq n
$$

are satisfied on $\Omega$;

5) $v: E \rightarrow R$ is a solution of (3), (4) and $v$ is of class $C^{1}$ on $E$ and there is a function $\alpha_{0}: \Delta \rightarrow R_{+}$such that

$$
\left|\varphi^{(r, m)}-\varphi_{h}^{(r, m)}\right| \leq \alpha_{0}(h) \quad \text { on } \quad E_{0, h} \cup \partial_{0} E_{h} \quad \text { and } \quad \lim _{h \rightarrow 0} \alpha_{0}(h)=0 ;
$$

6) $z_{h}: E_{h} \rightarrow R$ is a solution of (7), (8).

Then there is a function $\alpha: \Delta \rightarrow R_{+}$such that

$$
\left\|v_{h}-z_{h}\right\|_{r, h} \leq \alpha(h) \quad \text { for } \quad 0 \leq r \leq N_{0} \quad \text { and } \quad \lim _{h \rightarrow 0} \alpha(h)=0
$$

where $v_{h}$ is the restriction of $v$ to the set $E_{h}$.

The above theorem may be proved by a method used in [1-3]. Note that the Lipschitz condition (9) may be replaced in Theorem 1 by a nonlinear estimate of the Perron type.

Remark 1. Suppose that all the assumptions of Theorem 1 are satisfied and the solution $v: E \rightarrow R$ of (3), (4) is of class $C^{2}$.

Then there are $C_{0}, C \in R_{+}$such that we have the following error estimate for method (7), (8):

$$
\left\|v_{h}-z_{h}\right\|_{r, h} \leq C_{0} \alpha_{0}(h)+C h_{0}, \quad 0 \leq r \leq N_{0}
$$

The above result may be proved by methods used in [1-3].

Note that the classical Euler method [1,2] is not applicable to problem (3), (4). Now we formulate a new class of difference problems corresponding to (3), (4). We need next assumptions on $f$.

Assumption $\boldsymbol{H}_{\mathbf{0}}[\boldsymbol{f}]$. Suppose that $f \in C(\Omega, R)$ and

1) the partial derivatives

$$
\begin{gathered}
\left(\partial_{x_{1}} f(P), \ldots, \partial_{x_{n}} f(P)\right)=\partial_{x} f(P), \partial_{p} f(P), \\
\left(\partial_{q_{1}} f(P), \ldots, \partial_{q_{n}} f(P)\right)=\partial_{q} f(P)
\end{gathered}
$$

exist for $P=(t, x, p, q) \in \Omega$ and $\partial_{x} f, \partial_{q} f \in C\left(\Omega, R^{n}\right), \partial_{p} f \in C(\Omega, R)$;

$2)$ conditions (1), (2) are satisfied.

Write

$$
E_{h}^{\prime}=\left\{\left(t^{(r)}, x^{(m)}\right): 0 \leq r \leq N_{0}-1,-N<m<N\right\}
$$


Let us denote by $(z, u), u=\left(u_{1}, \ldots, u_{n}\right)$, the unknown functions of the variables $\left(t^{(r)}, x^{(m)}\right)$. Write

$$
\begin{gathered}
P^{(r, m)}[z, u]=\left(t^{(r)}, x^{(m)}, z^{(r, m)}, u^{(r, m)}\right), \\
Q^{(r, m)}[z, u]=\left(t^{(r)}, x^{(m)}, z^{(r, m)}, u^{(r+1, m)}\right) .
\end{gathered}
$$

We consider the quasilinear system of difference equations

$$
\delta_{0} z^{(r, m)}=f\left(Q^{(r, m)}[z, u]\right)+\sum_{j=1}^{n} \partial_{q_{j}} f\left(Q^{(r, m)}[z, u]\right)\left(\delta_{j} z^{(r, m)}-u_{j}^{(r, m)}\right)
$$

and

$$
\begin{aligned}
\delta_{0} u_{i}^{(r, m)}= & \partial_{x_{i}} f\left(P^{(r, m)}[z, u]\right)+\partial_{p} f\left(P^{(r, m)}[z, u]\right) u_{i}^{(r, m)}+ \\
& +\sum_{j=1}^{n} \partial_{q_{j}} f\left(P^{(r, m)}[z, u]\right) \delta_{j} u_{i}^{(r, m)}, \quad 1 \leq i \leq n,
\end{aligned}
$$

with the initial boundary condition

$$
z^{(r, m)}=\varphi_{h}^{(r, m)} \quad \text { and } \quad u^{(r, m)}=\psi_{h}^{(r, m)} \quad \text { on } \quad E_{0, h} \cup \partial_{0} E_{h},
$$

where $\varphi_{h}: E_{0, h} \cup \partial_{0} E_{h} \rightarrow R$ and $\psi_{h}: E_{0, h} \cup \partial_{0} E_{h} \rightarrow R^{n}$ are given functions. The difference operators $\delta_{0}$ and $\delta=\left(\delta_{1}, \ldots, \delta_{n}\right)$ are defined in the following way. Suppose that the functions $(z, u)$ are given on the sets $E_{r, h}$ and $E_{r+1, h}$, respectively, where $0 \leq r<N_{0}$. Then we put

$$
\delta_{0} z^{(r, m)}=\frac{1}{h_{0}}\left(z^{(r+1, m)}-z^{(r, m)}\right), \quad \delta_{0} u_{i}^{(r, m)}=\frac{1}{h_{0}}\left(u_{i}^{(r+1, m)}-u_{i}^{(r, m)}\right), \quad 1 \leq i \leq n .
$$

The difference operators with respect to spatial variables are defined in the following way:

$$
\begin{gathered}
\text { if } \partial_{q_{j}} f\left(Q^{(r, m)}[z, u]\right) \geq 0 \text { then } \delta_{j} z^{(r, m)}=\frac{1}{h_{j}}\left(z^{\left(r, m+e_{j}\right)}-z^{(r, m)}\right), \\
\text { if } \partial_{q_{j}} f\left(Q^{(r, m)}[z, u]\right)<0 \text { then } \delta_{j} z^{(r, m)}=\frac{1}{h_{j}}\left(z^{(r, m)}-z^{\left(r, m-e_{j}\right)}\right), \\
\text { if } \partial_{q_{j}} f\left(P^{(r, m)}[z, u]\right) \geq 0 \text { then } \delta_{j} u_{i}^{(r, m)}=\frac{1}{h_{j}}\left(u_{i}^{\left(r, m+e_{j}\right)}-u_{i}^{(r, m)}\right), \quad 1 \leq i \leq n, \\
\text { if } \partial_{q_{j}} f\left(P^{(r, m)}[z, u]\right)<0 \text { then } \delta_{j} u_{i}^{(r, m)}=\frac{1}{h_{j}}\left(u_{i}^{(r, m)}-u_{i}^{\left(r, m-e_{j}\right)}\right), \quad 1 \leq i \leq n .
\end{gathered}
$$


The difference problem consisting of system of difference equations (10), (11) with $\delta_{0}$ and $\delta$ given by (13) - (17) and initial boundary condition (12) is called a generalized Euler method for problem (3), (4). There exists exactly one solution $\left(z_{h}, u_{h}\right): E_{h} \rightarrow R^{1+n}, u_{h}=\left(u_{h, 1}, \ldots, u_{h, n}\right)$, of the above difference problem. For this purpose note that if $\left(z_{h}, u_{h}\right)$ are defined on $E_{r, h}$, $0 \leq r<N_{0}$, and $\left(t^{(r+1)}, x^{(m)}\right) \in E_{h}^{\prime}$ then

$$
\left(t^{(r)}, x^{\left(m+e_{j}\right)}\right), \quad \text { and }\left(t^{(r)}, x^{\left(m-e_{j}\right)}\right) \in E_{h} \quad \text { for } \quad 1 \leq j \leq n
$$

It follows that $u_{h}^{(r+1, m)}$ may be calculated from (11), (12) and then $z^{(r+1, m)}$ may be calculated from (10), (12). Then by induction the solution exists and it is unique on $E_{h}$.

There are two main differences between the classical result presented in Theorem 1 and our methods.

1. If we apply difference problem (7), (8) then we approximate the spatial derivatives of $z$ in (3) with the use of difference expressions (6). In our method we approximate the derivatives with respect to $x$ by using solutions of difference quations (11) which are generated by the original problem.

2. Suppose that we calculate the number $z_{h}^{(r+1, m)}$ by using method (7), (8). Then we apply the vector $\delta z$ considered at the point $\left(t^{(r)}, x^{(m)}\right)$. In our method we calculate $z_{h}^{(r+1, m)}$ by means of (10) and we need the vector $u_{h}$ at the point $\left(t^{(r)}, x^{(m)}\right)$ and also at the point $\left(t^{(r+1)}, x^{(m)}\right)$.

Difference problem (10)-(12) is obtained in the following way. Suppose that Assumption $H_{0}[f]$ is satisfied and that the function $\varphi: E_{0} \cup \partial_{0} E \rightarrow R$ is of class $C^{1}$. Existence theory for classical or generalized solutions to mixed problem (3), (4) is based on a method of quasilinearization. The method consists in replacing problem (3), (4) with the following quasilinear differential system for unknown functions $(z, u), u=\left(u_{1}, \ldots, u_{n}\right)$, of the variables $(t, x)$ :

$$
\begin{aligned}
\partial_{t} z(t, x)= & f(U[z, u ; t, x])+ \\
& +\sum_{j=1}^{n} \partial_{q_{j}} f(U[z, u ; t, x])\left(\partial_{x_{j}} z(t, x)-u_{j}(t, x)\right)
\end{aligned}
$$

and

$$
\begin{aligned}
\partial_{t} u_{i}(t, x)= & \partial_{x_{i}} f(U[z, u ; t, x])+\partial_{p} f(U[z, u ; t, x]) u_{i}(t, x)+ \\
& +\sum_{j=1}^{n} \partial_{q_{j}} f(U[z, u ; t, x]) \partial_{x_{j}} u_{i}(t, x), \quad 1 \leq i \leq n,
\end{aligned}
$$

with the initial boundary condition

$$
z(t, x)=\varphi(t, x) \quad \text { and } \quad u(t, x)=\partial_{x} \varphi(t, x) \quad \text { for } \quad(t, x) \in E_{0} \cup \partial_{0} E
$$

where $U[z, u ; t, x]=(t, x, z(t, x), u(t, x))$. Note that each equation of system (18), (19) depends on the unknown functions $(z, u)$ and it contains partial derivatives of only one scalar function. 
Under natural assumptions on given functions, the following properties of problem (18)(20) may be proved $([4,5])$ :

(A) If $(\tilde{z}, \tilde{u}): E \rightarrow R^{1+n}, \tilde{u}=\left(\tilde{u}_{1}, \ldots, \tilde{u}_{n}\right)$, is a classical solution of (18) $-(20)$ then $\partial_{x} \tilde{z}=\tilde{u}$ on $E$.

(B) If $\tilde{v}: E \rightarrow R$ is a solution of (3), (4) and $v$ is of class $C^{2}$ on $E$ then the functions $\left(\tilde{v}, \partial_{x} \tilde{v}\right)$ satisfy $(18)-(20)$.

(C) If $\left(\tilde{z}, \partial_{x} \tilde{z}\right): E \rightarrow R^{1+n}$ is a solution of (18) - (20) then $\tilde{z}$ satisfies (3), (4).

Difference problem (10) - (12) is a discretization of (18) - (20). The principal significance of system (10), (11) is that the method of discretization of (18), (19) depends on the properties of the functions $\left(\partial_{q_{1}} f, \ldots, \partial_{q_{n}} f\right)$ and on the previous values of the unknown functions. System (18), (19) has the following property: the differential equations of bicharacteristics for (18) and for (19) are the same and they have the form

$$
\eta^{\prime}(t)=-\partial_{q} f(t, \eta(t), z(t, \eta(t)), u(t, \eta(t)))
$$

This property of system (18), (19) is important in the investigation of the stability of difference problem (10) - (17).

Remark 2. Suppose that $f \in C(\Omega, R)$ and

1) the derivatives $\left(\partial_{q_{1}} f, \ldots, \partial_{q_{n}} f\right)=\partial_{q} f$ exist on $\Omega$ and $\partial_{q} f \in C\left(\Omega, R^{n}\right)$;

2) the Lipschitz condition (9) is satisfied and conditions (1), (2) hold.

Then the classical solution of mixed problem (3), (4) is unique. This result may be proved by a method of differential inequalities.

Note that condition (9) may be replaced in the above statement by a nonlinear estimate of the Perron type.

Existence results for mixed problem (3), (4) may be deduced from [4, 5], see also [6].

The papers [1-3] initiated the investigations of difference methods for nonlinear partial differential equations and weakly coupled systems. Initial problems on the Haar pyramid and initial boundary-value problems were considered. The main question in the theory of numerical methods for nonlinear differential equations is to find a difference equation generated by the original problem, which is stable. The method of difference inequalities or theorems on linear recurrent inequalities are used in the investigation of the stability. The monograph [7] contains an exposition of recent developments on difference methods for hyperbolic differential or functional differential equations.

The paper is organized as follows. In Section 2 we give sufficient conditions for the convergence of the generalized Euler method. In the next section we consider some modification of method (10)-(12) and we prove a convergence result under assumptions that the derivatives $\partial_{x} f, \partial_{p} f, \partial_{q} f$ satisfy nonlinear estimates of the Perron type with respect to variables $(p, q)$. Numerical examples are given in the last part of the paper. Our results are a continuation of paper [8], where the generalized Euler method was considered for initial problems on the Haar pyramid. We use in the paper general ideas concerning difference equations which were introduced in $[2,9,10]$.

2. Convergence of the generalized Euler method. We formulate next assumptions on $f$. Assumption $\boldsymbol{H}[\boldsymbol{f}]$. Suppose that Assumption $H_{0}[f]$ is satisfied and 
1) there is $A \in R_{+}$such that

$$
\left\|\partial_{x} f(P)\right\|, \quad\left|\partial_{p} f(P)\right|,\left\|\partial_{q} f(P)\right\| \leq A
$$

for $P=(t, x, p, q) \in \Omega$;

2) there is $L \in R_{+}$such that the terms

$$
\begin{gathered}
\| \partial_{x} f(t, x, p, q)-\partial_{x} f\left(t, x, \bar{p}, \bar{q} \|, \quad \mid \partial_{p} f(t, x, p, q)-\partial_{p} f(t, x, \bar{p}, \bar{q} \mid,\right. \\
\| \partial_{q} f(t, x, p, q)-\partial_{q} f(t, x, \bar{p}, \bar{q} \|
\end{gathered}
$$

are bounded from above by $L(|p-\bar{p}|+\|q-\bar{q}\|)$.

We prove the main result on the generalized Euler method.

Theorem 2. Suppose that Assumption $H[f]$ is satisfied and

1) $h \in \Delta$ and

$$
1-h_{0} \sum_{j=1}^{n} \frac{1}{h_{j}}\left|\partial_{q_{j}} f(t, x, p, q)\right| \geq 0 \quad \text { on } \quad \Omega
$$

2) the function $\varphi: E_{0} \cup \partial_{0} E \rightarrow R$ is of class $C^{2}$ and $v: E \rightarrow R$ is a solution of problem (3), (4) and $v$ is of class $C^{2}$ on $E$;

3) the functions $\left(z_{h}, u_{h}\right): E_{h} \rightarrow R^{1+n}$, where $u_{h}=\left(u_{h, 1}, \ldots, u_{h, n}\right)$, satisfy (10)-(12) with $\delta_{0}$ and $\delta$ given by (13) - (17) and there is a function $\alpha_{0}: \Delta \rightarrow R_{+}$such that

$$
\left|\varphi^{(r, m)}-\varphi_{h}^{(r, m)}\right|+\left\|\partial_{x} \varphi^{(r, m)}-\psi_{h}^{(r, m)}\right\| \leq \alpha_{0}(h) \quad \text { on } \quad E_{0, h} \cup \partial_{0} E_{h}
$$

and $\lim _{h \rightarrow 0} \alpha_{0}(h)=0$.

$\stackrel{h \rightarrow 0}{\text { Then }}$ there is a function $\alpha: \Delta \rightarrow R_{+}$such that

$$
\left\|v_{h}-z_{h}\right\|_{r, h}+\left\|\partial_{x} v_{h}-u_{h}\right\|_{r, h} \leq \alpha(h), \quad 0 \leq r \leq N_{0},
$$

and $\lim _{h \rightarrow 0} \alpha(h)=0$, where $v_{h}$ and $\partial_{x} v_{h}$ are the restrictions of $v$ and $\partial_{x} v$ respectively to the set $E_{h}$.

Proof. Write $w=\partial_{x} v$ and $w=\left(w_{1}, \ldots, w_{n}\right)$. Let us denote by $w_{h}=\left(w_{h, 1}, \ldots, w_{h, n}\right)$ the restriction of $w$ to the set $E_{h}$ and put

$$
\xi_{h}=v_{h}-z_{h}, \quad \lambda_{h}=w_{h}-u_{h}
$$

with $\lambda_{h}=\left(\lambda_{h, 1}, \ldots, \lambda_{h, n}\right)$. Let the functions $\omega_{h, 0}, \omega_{h, 1}: I_{h} \rightarrow R$ be defined by

$$
\omega_{h, 0}^{(r)}=\left\|\xi_{h}\right\|_{r, h}, \quad \omega_{h, 1}^{(r)}=\left\|\lambda_{h}\right\|_{r, h},
$$

where $0 \leq r \leq N_{0}$. Set $\omega_{h}=\omega_{h, 0}+\omega_{h, 1}$. We will write a difference inequality for the function $\omega_{h}$. We first prove some properties of $\omega_{h, 0}$. Let the functions $\Gamma_{h, 0}, \Lambda_{h, 0}: E_{h}^{\prime} \rightarrow R$ be defined by

$$
\Gamma_{h, 0}^{(r, m)}=\delta_{0} v_{h}^{(r, m)}-\partial_{t} v^{(r, m)}+\sum_{j=1}^{n} \partial_{q_{j}} f\left(P^{(r, m)}[v, w]\right)\left(\partial_{x_{j}} v^{(r, m)}-\delta_{j} v_{h}^{(r, m)}\right)
$$


and

$$
\begin{aligned}
\Lambda_{h, 0}^{(r, m)}= & f\left(P^{(r, m)}[v, w]\right)-f\left(Q^{(r, m)}\left[z_{h}, u_{h}\right]\right)+ \\
& +\sum_{j=1}^{n} \partial_{q_{j}} f\left(Q^{(r, m)}\left[z_{h}, u_{h}\right]\right) u_{h, j}^{(r, m)}- \\
& -\sum_{j=1}^{n} \partial_{q_{j}} f\left(P^{(r, m)}[v, w]\right) w_{j}^{(r, m)}+ \\
& +\sum_{j=1}^{n}\left\{\partial_{q_{j}} f\left(P^{(r, m)}[v, w]\right)-\partial_{q_{j}} f\left(Q^{(r, m)}\left[z_{h}, u_{h}\right]\right)\right\} \delta_{j} v_{h}^{(r, m)} .
\end{aligned}
$$

Put

$$
W_{h}\left[\xi_{h}\right]^{(r, m)}=\xi_{h}^{(r, m)}+h_{0} \sum_{j=1}^{n} \partial_{q_{j}} f\left(Q^{(r, m)}\left[z_{h}, u_{h}\right]\right) \delta_{j} \xi_{h}^{(r, m)}
$$

It follows from (10) and (18) that $\xi_{h}$ satisfies the difference equation

$$
\xi_{h}^{(r+1, m)}=W_{h}\left[\xi_{h}\right]^{(r, m)}+h_{0}\left[\Gamma_{h, 0}^{(r, m)}+\Lambda_{h, 0}^{(r, m)}\right]
$$

where $\left(t^{(r)}, x^{(m)}\right) \in E_{h}^{\prime}$. Write

$$
\begin{gathered}
J_{+}[r, m]=\left\{j \in\{1, \ldots, n\}: \partial_{q_{j}} f\left(Q^{(r, m)}\left[z_{h}, u_{h}\right]\right) \geq 0\right\}, \\
J_{-}[r, m]=\{1, \ldots, n\} \backslash J_{+}[r, m] .
\end{gathered}
$$

It follows from the definition of the operator $\delta$ that the function $\xi_{h}$ satisfies the relation

$$
\begin{aligned}
W_{h}\left[\xi_{h}\right]^{(r, m)}= & \xi_{j}^{(r, m)}\left[1-h_{0} \sum_{j=1}^{n} \frac{1}{h_{j}} \mid \partial_{q_{j}} f\left(Q^{(r, m)}\left[z_{h}, u_{h}\right]\right)\right]+ \\
& +h_{0} \sum_{j \in J_{+}[r, m]} \frac{1}{h_{j}} \partial_{q_{j}} f\left(Q^{(r, m)}\left[z_{h}, u_{h}\right]\right) \xi_{h}^{\left(r, m+e_{j}\right)}- \\
& -h_{0} \sum_{j \in J_{-}[r, m]} \frac{1}{h_{j}} \partial_{q_{j}} f\left(Q^{(r, m)}\left[z_{h}, u_{h}\right]\right) \xi_{h}^{\left(r, m-e_{j}\right)}
\end{aligned}
$$


It follows from assumptions (1), (2), (21) and from (6) that

$$
\left|W_{h}\left[\xi_{h}\right]^{(r, m)}\right| \leq \omega_{h, 0}^{(r)} \quad \text { on } \quad E_{h}^{\prime}
$$

Since the functions $(v, w)$ satisfy equation (18), it follows that there is $\gamma_{0}: \Delta \rightarrow R_{+}$such that

$$
\left|\Gamma_{h, 0}^{(r, m)}\right| \leq \gamma_{0}(h) \quad \text { on } \quad E_{h}^{\prime} \quad \text { and } \quad \lim _{h \rightarrow 0} \gamma_{0}(h)=0
$$

Let $\tilde{c} \in R_{+}$be such a constant that

$$
\begin{gathered}
\left|\partial_{t} v(t, x)\right|, \quad\left\|\partial_{x} v(t, x)\right\|, \\
\left\|\partial_{t x} v(t, x)\right\|, \quad\left\|\partial_{x x} v(t, x)\right\| \leq \tilde{c} \text { on } E
\end{gathered}
$$

where

$$
\partial_{x x} v(t, x)=\left[\partial_{x_{i} x_{j}} v(t, x)\right]_{i, j=1, \ldots, n}
$$

and

$$
\left\|\partial_{x x} v(t, x)\right\|=\max \left\{\sum_{i=1}^{n}\left|\partial_{x_{i} x_{j}} v(t, x)\right|: 1 \leq j \leq n\right\} .
$$

According to Assumption $H[f]$ we have

$$
\left|\Lambda_{h, 0}^{(r, m)}\right| \leq A \omega_{h, 1}^{(r)}+(A+2 \tilde{c} L)\left[\omega_{h, 0}^{(r)}+\omega_{h, 1}^{(r+1)}+\tilde{c} h_{0}\right]
$$

where $\left(t^{(r)}, x^{(m)}\right) \in E_{h}^{\prime}$. Applying (28)-(32) we conclude that the recurrent inequality

$$
\begin{aligned}
\omega_{h, 0}^{(r+1)} \leq & \omega_{h, 0}^{(r)}+A h_{0} \omega_{h, 1}^{(r)}+h_{0}(A+2 \tilde{c} L)\left[\omega_{h, 0}^{(r)}+\omega_{h, 1}^{(r+1)}\right]+ \\
& +h_{0}\left[\gamma_{0}(h)+h_{0} \tilde{c}(A+2 \tilde{c} L)\right]
\end{aligned}
$$

is satisfied for $0 \leq r \leq N_{0}-1$.

Now we write a difference inequality for $\omega_{h, 1}$. Let the functions

$$
\begin{gathered}
\Gamma_{h}=\left(\Gamma_{h, 1}, \ldots, \Gamma_{h, n}\right): E_{h}^{\prime} \rightarrow R^{n}, \\
\Lambda_{h}=\left(\Lambda_{h, 1}, \ldots, \Lambda_{h, n}\right): E_{h}^{\prime} \rightarrow R^{n}
\end{gathered}
$$


be defined by

$$
\begin{aligned}
\Gamma_{h, i}^{(r, m)}= & \delta_{0} w_{h, i}^{(r, m)}-\partial_{t} w_{i}^{(r, m)}+ \\
& +\sum_{j=1}^{n} \partial_{q_{j}} f\left(P^{(r, m)}[v, w]\right)\left(\partial_{x_{j}} w_{i}^{(r, m)}-\delta_{j} w_{h, i}^{(r, m)}\right), \quad 1 \leq i \leq n,
\end{aligned}
$$

and

$$
\begin{aligned}
\Lambda_{h, i} & =\partial_{x_{i}} f\left(P^{(r, m)}[v, w]\right)-\partial_{x_{i}} f\left(P^{(r, m)}\left[z_{h}, u_{h}\right]\right)+ \\
& +\partial_{p} f\left(P^{(r, m)}[v, w]\right) w_{i}^{(r, m)}-\partial_{p} f\left(P^{(r, m)}\left[z_{h}, u_{h}\right]\right) u_{h, i}^{(r, m)}+ \\
& +\sum_{j=1}^{n}\left[\partial_{q_{j}} f\left(P^{(r, m)}[v, w]\right)-\partial_{q_{j}} f\left(P^{(r, m)}\left[z_{h}, u_{h}\right]\right)\right] \delta_{j} w_{h, i}^{(r, m)}, \quad 1 \leq i \leq n .
\end{aligned}
$$

Write

$$
\begin{aligned}
\widetilde{U}_{h}\left[\lambda_{h, i}\right]^{(r, m)}= & \lambda_{h, i}^{(r, m)}+ \\
& +h_{0} \sum_{j=1}^{n} \partial_{q_{j}} f\left(P^{(r, m)}\left[z_{h}, u_{h}\right]\right) \delta_{j} \lambda_{h, i}^{(r, m)}, \quad 1 \leq i \leq n,
\end{aligned}
$$

and

$$
U_{h}\left[\lambda_{h}\right]^{(r, m)}=\left(\widetilde{U}_{h}\left[\lambda_{h, 1}\right]^{(r, m)}, \ldots, \widetilde{U}_{h}\left[\lambda_{h, n}\right]^{(r, m)}\right)
$$

It follows from (11) and (19) that the function $\lambda_{h}$ satisfies the difference equation

$$
\lambda_{h}^{(r, m)}=U_{h}\left[\lambda_{h}\right]^{(r, m)}+h_{0}\left[\Gamma_{h}^{(r, m)}+\Lambda_{h}^{(r, m)}\right]
$$

where $\left(t^{(r)}, x^{(m)}\right) \in E_{h}^{\prime}$. Write

$$
\begin{gathered}
I_{+}[r, m]=\left\{j \in\{1, \ldots, n\}: \partial_{q_{j}} f\left(P^{(r, m)}\left[z_{h}, u_{h}\right]\right) \geq 0\right\}, \\
I_{-}[r, m]=\{1, \ldots, n\} \backslash I_{+}[r, m] .
\end{gathered}
$$


Then we have

$$
\begin{aligned}
\widetilde{U}_{h}\left[\lambda_{h, i}\right]^{(r, m)}= & \lambda_{h, i}^{(r, m)}\left[1-h_{0} \sum_{j=1}^{n} \frac{1}{h_{j}}\left|\partial_{q_{j}} f\left(P^{(r, m)}\left[z_{h}, u_{h}\right]\right)\right|\right]+ \\
& +h_{0} \sum_{j \in I_{+}[r, m]} \frac{1}{h_{j}} \partial_{q_{j}} f\left(P^{(r, m)}\left[z_{h}, u_{h}\right]\right) \lambda_{h, i}^{\left(r, m+e_{j}\right)}- \\
& -h_{0} \sum_{j \in I_{-}[r, m]} \frac{1}{h_{j}} \partial_{q_{j}} f\left(P^{(r, m)}\left[z_{h}, u_{h}\right]\right) \lambda_{h, i}^{\left(r, m-e_{j}\right)}, \quad 1 \leq i \leq n .
\end{aligned}
$$

According to (1), (2), (21) and (6), we have

$$
\left\|U_{h}\left[\lambda_{h}\right]^{(r, m)}\right\| \leq \omega_{h, 1}^{(r)} \quad \text { on } \quad E_{h}^{\prime}
$$

Since the functions $(v, w)$ satisfy equations (19), it follows that there is $\gamma: \Delta \rightarrow R_{+}$such that

$$
\left\|\Gamma_{h}^{(r, m)}\right\| \leq \gamma(h) \quad \text { on } \quad E_{h}^{\prime} \quad \text { and } \quad \lim _{h \rightarrow 0} \gamma(h)=0
$$

It follows from Assumption $H[f]$ that

$$
\left\|\Lambda_{h}^{(r, m)}\right\| \leq L \omega_{h}^{(r)}(1+2 \tilde{c})+A \omega_{h, 1}^{(r)} \quad \text { on } \quad E_{h}^{\prime},
$$

and consequently

$$
\omega_{h, 1}^{(r+1)} \leq \omega_{h, 1}^{(r)}+h_{0} \gamma(h)+L h_{0}(1+2 \tilde{c}) \omega_{h}^{(r)}+A h_{0} \omega_{h, 1}^{(r)}
$$

where $0 \leq r \leq N_{0}-1$. Combining (33) with (39) we see that there are $\widetilde{L}>0$ and $\tilde{\gamma}: \Delta \rightarrow R_{+}$ such that

$$
\omega_{h}^{(r+1)} \leq \omega_{h}^{(r)}\left(1+\tilde{L} h_{0}\right)+h_{0} \tilde{\gamma}(h), \quad 0 \leq r \leq N_{0}-1,
$$

and $\lim _{h \rightarrow 0} \tilde{\gamma}(h)=0$ which implies that

$$
\omega_{h}^{(r)} \leq \alpha_{0}(h) e^{\tilde{L} a}+\tilde{\gamma}(h) \frac{e^{\tilde{L} a}-1}{\widetilde{L}}, \quad 0 \leq r \leq N_{0} .
$$

This completes the proof.

Remark 3. Suppose that all the assumptions of Theorem 2 are satisfied and there is $M \in R_{+}^{n}$ such that $h^{\prime} \leq M h_{0}$. Then there are $C_{0}, C \in R_{+}$such that we have the following error estimate for method (10) - (12): 


$$
\left\|v_{h}-z_{h}\right\|_{r, h}+\left\|\partial_{x} v_{h}-u_{h}\right\|_{r, h} \leq C_{0} \alpha_{0}(h)+C h_{0}
$$

where $0 \leq r \leq N_{0}$.

The above result is a consequence of (30), (38) and (40).

Remark 4. In the results on error estimates we need estimates for the derivatives of the solution $v$ of problem (3), (4). One may obtain them by the method of differential inequalities. The results given in [11] (Chapter VII ) and [12] for initial problems on the Haar pyramid can be easily extended to mixed problems.

3. Convergence of the difference method with nonlinear estimates. We prove that the generalized Euler method is convergent if the Lipschitz condition for the derivatives $\partial_{x} f, \partial_{p} f, \partial_{q} f$ is replaced by a nonlinear estimate of the Perron type. We change equation (10) in this case. We consider the quasilinear system of difference equations consisting of equations (11) and

$$
\delta_{0} z^{(r, m)}=f\left(P^{(r, m)}[z, u]\right)+\sum_{j=1}^{n} \partial_{q_{j}} f\left(P^{(r, m)}[z, u]\right)\left(\delta_{j} z^{(r, m)}-u_{j}^{(r, m)}\right)
$$

with initial boundary condition (12). Difference expressions

$$
\delta_{0} z^{(r, m)} \quad \text { and } \quad \delta_{0} u_{i}^{(r, m)}, \quad \delta u_{i}^{(r, m)}=\left(\delta_{1} u_{i}^{(r, m)}, \ldots, \delta_{n} u_{i}^{(r, m)}\right), \quad 1 \leq i \leq n,
$$

are given by (13) and (16), (17), respectively. Difference operator $\delta z^{(r, m)}$ is given by (14), (15) with $P^{(r, m)}[z, u]$ instead of $Q^{(r, m)}[z, u]$. Numerical results obtained by the above difference, method are better than those obtained by (7), (8). We show a numerical example.

Our basic assumptions are the following.

Assumption $\boldsymbol{H}[\boldsymbol{\sigma}]$. The function $\sigma:[0, a] \times R_{+} \rightarrow R_{+}$is continuous and

1) $\sigma$ is nondecreasing with respect to both variables and $\sigma(t, 0)=0$ for $t \in[0, a]$;

2) for each $c \in R_{+}$and $d \geq 1$ the maximal solution of the Cauchy problem

$$
\eta^{\prime}(t)=c \eta(t)+d \sigma(t, \eta(t)), \quad \eta(0)=0
$$

is $\eta(t)=0, t \in[0, a]$.

Assumption $\tilde{\boldsymbol{H}}[\boldsymbol{f}]$. Suppose that Assumption $H_{0}[f]$ and condition 1 of Assumption $H[f]$ are satisfied and there is a function $\sigma:[0, a] \times R_{+} \rightarrow R_{+}$satisfying Assumption $H[\sigma]$ and such that the terms

$$
\begin{gathered}
\left\|\partial_{x} f(t, x, p, q)-\partial_{x} f(t, x, \bar{p}, \bar{q})\right\|, \quad\left|\partial_{p} f(t, x, p, q)-\partial_{p} f(t, x, \bar{p}, \bar{q})\right|, \\
\left\|\partial_{q} f(t, x, p, q)-\partial_{q} f(t, x, \bar{p}, \bar{q})\right\|
\end{gathered}
$$

are bounded from above by $\sigma(t,|p-\bar{p}|+\|q-\bar{q}\|)$.

Theorem 3. Suppose that Assumption $\widetilde{H}[f]$ is satisfied and 
1) $h \in \Delta$ and condition (21) holds;

2) the function $\varphi: E_{0} \cup \partial_{0} E \rightarrow R$ is of class $C^{2}$ and $v: E \rightarrow R$ is a solution of problem (3), (4) and $v$ is of class $C^{2}$ on $E$;

3) the function $\left(z_{h}, u_{h}\right): E_{h} \rightarrow R^{1+n}, u_{h}=\left(u_{h, 1}, \ldots, u_{h, n}\right)$, satisfies (11), (41) and (12);

4) there is a function $\alpha_{0}: \Delta \rightarrow R_{+}$such that initial boundary estimate (22) is satisfied and $\lim _{h \rightarrow 0} \alpha_{0}(h)=0$.

Then there is a number $\varepsilon_{0}>0$ and a function $\alpha: \Delta \rightarrow R_{+}$such that condition (23) is satisfied for $\|h\|<\varepsilon_{0}$ and $\lim _{h \rightarrow 0} \alpha(h)=0$.

Proof. Write $w=\partial_{x} v$. Let $\xi: E_{h} \rightarrow R$ and $\lambda_{h}: E_{h} \rightarrow R^{n}$ be the functions defined by (24) where $v_{h}$ and $w_{h}$ are the restrictions of $v$ and $w$ to the mesh $E_{h}$. We can now proceed analogously to the proof of Theorem 2 . It follows easily that the functions $\omega_{h, 0}$ and $\omega_{h, 1}$ given by (25) satisfy the recurrent inequalities

$$
\omega_{h, 0}^{(r+1)} \leq \omega_{h, 0}^{(r)}+2 A h_{0} \omega_{h}^{(r)}+2 \tilde{c} h_{0} \sigma\left(t^{(r)}, \omega_{h}^{(r)}\right)+h_{0} \gamma_{0}(h)
$$

and

$$
\omega_{h, 1}^{(r+1)} \leq \omega_{h, 1}^{(r)}+A h_{0} \omega_{h}^{(r)}+(1+2 \tilde{c}) \sigma\left(t^{(r)}, \omega_{h}^{(r)}\right)+h_{0} \gamma(h)
$$

where $0 \leq r \leq N_{0}-1$ and $\omega_{h}=\omega_{h, 0}+\omega_{h, 1}$. The functions $\gamma_{0}$ and $\gamma$ are given by conditions (30) and (38) respectively, the constant $\tilde{c}$ is such that estimates (31) hold. The functions $\Gamma_{h, 0}$, $\Gamma_{h}$ and $\Lambda_{h}$ are defined by (26), (34) and (35) respectively, whereas the function $\Lambda_{h, 0}$ is given by (27) with $P^{(r, m)}\left[z_{h}, u_{h}\right]$ instead of $Q^{(r, m)}\left[z_{h}, u_{h}\right]$. Adding inequalities (42) and (43) we conclude that the function $\omega_{h}$ satisfies the recurrent inequality

$$
\omega_{h}^{(r+1)} \leq \omega_{h}^{(r)}+c h_{0} \omega_{h}^{(r)}+d h_{0} \sigma\left(t^{(r)}, \omega_{h}^{(r)}\right)+h_{0}\left(\gamma_{0}(h)+\gamma(h)\right), \quad 0 \leq r \leq N_{0}-1,
$$

where $c=3 A, d=1+4 \tilde{c}$. Consider the Cauchy problem

$$
\eta^{\prime}(t)=c \eta(t)+d \sigma(t, \eta(t))+\gamma_{0}(h)+\gamma(h), \quad \eta(0)=\alpha_{0}(h) .
$$

It follows from Assumption $H[\sigma]$ that there is $\varepsilon_{0}>0$ such that for $\|h\|<\varepsilon_{0}$ there exists the maximum solution $\eta_{h}$ of the above problem and $\eta_{h}$ is defined on $[0, a]$. Moreover we have

$$
\lim _{h \rightarrow 0} \eta_{h}(t)=0 \quad \text { uniformly on } \quad[0, a] .
$$

The function $\eta_{h}$ satisfies the recurrent inequality

$$
\eta_{h}^{(r+1)} \geq \eta_{h}^{(r)}+c h_{0} \eta_{h}^{(r)}+d h_{0} \sigma\left(t^{(r)}, \eta_{h}^{(r)}\right)+h_{0}\left(\gamma_{0}(h)+\gamma(h)\right), \quad 0 \leq r \leq N_{0}-1
$$

Since $\omega_{h}^{(0)} \leq \eta_{h}^{(0)},(44)$ and (45) show that $\omega_{h}^{(r)} \leq \eta_{h}^{(r)}$ for $0 \leq r \leq N_{0}$. Then we get (23) with $\alpha(h)=\eta_{h}(a)$ for $\|h\|<\varepsilon_{0}$. This proves the theorem.

Remark 5. The results of the paper can be extended to weakly coupled nonlinear systems with initial boundary conditions. 
4. Numerical experiments. Now we give an example of a classical difference method and the generalized Euler method for a nonlinear equation.

Let $n=2$ and $E=[0,1] \times[-1,1] \times[1,-1]$. Denote by $z$ an unknown function of the variables $(t, x, y)$. Consider the differential equation

$$
\begin{aligned}
\partial_{t} z(t, x, y)= & x \partial_{x} z(t, x, y)+y \partial_{y} z(t, x, y)+ \\
& +\frac{1}{2} \sin \left[x \partial_{x} z(t, x, y)-y \partial_{y} z(t, x, y)\right]-4 z(t, x, y)+f(t, x, y),
\end{aligned}
$$

with the initial boundary condition

$$
z(t, x, y)=0 \quad \text { for } \quad(t, x, y) \in E_{0} \cup \partial_{0} E
$$

Here

$$
f(t, x, y)=\frac{1}{2} x y\left(x^{2}-1\right)\left(1-y^{2}\right)-t x y\left(1-x^{2} y^{2}\right)-\frac{1}{2} \sin \left[t x y\left(x^{2}-y^{2}\right)\right]
$$

The exact solution of this problem is known. It is

$$
w(t, x, y)=\frac{t}{2} x y\left(x^{2}-1\right)\left(1-y^{2}\right)
$$

Consider the classical difference equation for (46), (47)

$$
\begin{aligned}
z^{(r+1, m)}= & \frac{1}{4} z^{\left(r, m_{1}+1, m_{2}\right)}\left[1+2 x^{\left(m_{1}\right)} \frac{h_{0}}{h_{1}}\right]+ \\
& +\frac{1}{4} z^{\left(r, m_{1}-1, m_{2}\right)}\left[1-2 x^{\left(m_{1}\right)} \frac{h_{0}}{h_{1}}\right]+ \\
& +\frac{1}{4} z^{\left(r, m_{1}, m_{2}+1\right)}\left[1+2 y^{\left(m_{2}\right)} \frac{h_{0}}{h_{2}}\right]+ \\
& +\frac{1}{4} z^{\left(r, m_{1}, m_{2}-1\right)}\left[1-2 y^{\left(m_{2}\right)} \frac{h_{0}}{h_{2}}\right]+ \\
& +\frac{h_{0}}{2} \sin \left[A^{(r, m)}\right]-4 h_{0} z^{(r, m)}+h_{0} f^{(r, m)},
\end{aligned}
$$

where $0 \leq r \leq N_{0},-N \leq m \leq N, N=\left(N_{1}, N_{2}\right), m=\left(m_{1}, m_{2}\right)$, and the initial boundary condition

$$
z^{(r, m)}=0 \quad \text { for } \quad\left(t^{(r)}, x^{\left(m_{1}\right)}, y^{\left(m_{2}\right)}\right) \in E_{0, h} \cup \partial_{0} E_{h}
$$


where

$$
\begin{aligned}
A^{(r, m)}= & x^{\left(m_{1}\right)}\left(z^{\left(r, m_{1}+1, m_{2}\right)}-z^{\left(r, m_{1}-1, m_{2}\right)}\right)\left(2 h_{1}\right)^{-1}- \\
& -y^{\left(m_{2}\right)}\left(z^{\left(r, m_{1}, m_{2}+1\right)}-z^{\left(r, m_{1}, m_{2}-1\right)}\right)\left(2 h_{2}\right)^{-1} .
\end{aligned}
$$

Denote by $\tilde{z}_{h}: E_{h} \rightarrow R$ the solution of (48), (49).

Now we construct the generalized Euler method for problem (46), (47). Let us denote by $(z, u, v)$ unknown functions of the variables $\left(t^{(r)}, x^{\left(m_{1}\right)}, y^{\left(m_{2}\right)}\right)$. Write

$$
F\left(t, x, y, p, q_{1}, q_{2}\right)=x q_{1}+y q_{2}+\frac{1}{2} \sin \left(x q_{1}-y q_{2}\right)-4 p+f(t, x, y)
$$

and

$$
P^{(r, m)}=\left(t^{(r)}, x^{\left(m_{1}\right)}, y^{\left(m_{2}\right)}, z^{(r, m)}, u^{(r, m)}, v^{(r, m)}\right)
$$

Consider the system of difference equations

$$
\begin{aligned}
z^{(r+1, m)}= & z^{(r, m)}+h_{0} F\left(P^{(r, m)}\right)+ \\
& +h_{0} \partial_{q_{1}} F\left(P^{(r, m)}\right)\left(\delta_{1} z^{(r, m)}-u^{(r, m)}\right)+ \\
& +h_{0} \partial_{q_{1}} F\left(P^{(r, m)}\right)\left(\delta_{2} z^{(r, m)}-v^{(r, m)}\right),
\end{aligned}
$$

and

$$
\begin{aligned}
u^{(r+1, m)}= & u^{(r, m)}+h_{0} \partial_{x} F\left(P^{(r, m)}\right)+h_{0} \partial_{p} F\left(P^{(r, m)}\right) u^{(r, m)}+ \\
& +h_{0} \partial_{q_{1}} F\left(P^{(r, m)}\right) \delta_{1} u^{(r, m)}+h_{0} \partial_{q_{2}} F\left(P^{(r, m)}\right) \delta_{2} u^{(r, m)}, \\
v^{(r+1, m)}= & v^{(r, m)}+h_{0} \partial_{y} F\left(P^{(r, m)}\right)+h_{0} \partial_{p} F\left(P^{(r, m)}\right) v^{(r, m)}+ \\
& +h_{0} \partial_{q_{1}} F\left(P^{(r, m)}\right) \delta_{1} v^{(r, m)}+h_{0} \partial_{q_{2}} F\left(P^{(r, m)}\right) \delta_{2} v^{(r, m)},
\end{aligned}
$$

with the initial boundary conditions

$$
\begin{aligned}
& z^{(r, m)}=0 \quad \text { on } \quad E_{0, h} \cup \partial_{0} E_{h}, \\
& u^{(0, m)}=v^{(0, m)}=0 \quad \text { on } \quad E_{0, h}
\end{aligned}
$$


and

$$
\begin{gathered}
u^{\left(r, m_{1}, N_{2}\right)}=u^{\left(r, m_{1},-N_{2}\right)}=0, \\
v^{\left(r, N_{1}, m_{2}\right)}=v^{\left(r,-N_{1}, m_{2}\right)}=0, \\
u^{\left(r, N_{1}, m_{2}\right)}=u^{\left(r,-N_{1}, m_{2}\right)}=t^{(r)} y^{\left(m_{2}\right)}\left[1-\left(y^{\left(m_{2}\right)}\right)^{2}\right], \\
v^{\left(r, m_{1}, N_{2}\right)}=v^{\left(r, m_{1},-N_{2}\right)}=-t^{(r)} x^{\left(m_{1}\right)}\left[\left(x^{\left(m_{1}\right)}\right)^{2}-1\right],
\end{gathered}
$$

where $0 \leq r \leq N_{0},-N \leq m \leq N$. The difference operators

$$
\begin{gathered}
\left(\delta_{1} z^{(r, m)}, \delta_{2} z^{(r, m)}\right) \\
\left(\delta_{1} u^{(r, m)}, \delta_{2} u^{(r, m)}\right), \quad\left(\delta_{1} v^{(r, m)}, \delta_{2} v^{(r, m)}\right)
\end{gathered}
$$

are defined according to our theory.

Let us denote by $\left(z_{h}, u_{h}, v_{h}\right): E_{h} \rightarrow R^{3}$ the solution of the above difference problem. We give the following information on the errors of the methods. Write

$$
\begin{gathered}
\tilde{\eta}_{h}^{(r)}=\max \left\{\left|w^{(r, m)}-\tilde{z}_{h}^{(r, m)}\right|:-N \leq m \leq N\right\}, \\
\eta_{h}^{(r)}=\max \left\{\left|w^{(r, m)}-z_{h}^{(r, m)}\right|:-N \leq m \leq N\right\}, \quad 0 \leq r \leq N_{0},
\end{gathered}
$$

and

$$
\begin{gathered}
\tilde{\varepsilon}_{h}^{(r)}=\frac{1}{\left(2 N_{1}+1\right)\left(2 N_{2}+1\right)} \sum_{m=-N}^{N}\left|w^{(r, m)}-\tilde{z}_{h}^{(r, m)}\right|, \\
\varepsilon_{h}^{(r)}=\frac{1}{\left(2 N_{1}+1\right)\left(2 N_{2}+1\right)} \sum_{m=-N}^{N}\left|w^{(r, m)}-z_{h}^{(r, m)}\right|, \quad 0 \leq r \leq N_{0} .
\end{gathered}
$$

The numbers $\tilde{\eta}_{h}^{(r)}$ and $\eta_{h}^{(r)}$ are the maximal errors with fixed $t^{(r)}$. The numbers $\tilde{\varepsilon}_{h}^{(r)}$ and $\varepsilon_{h}^{(r)}$ are the arithmetical mean of the errors with fixed $t^{(r)}$. The values of the functions $\tilde{\eta}_{h}, \eta_{h}, \tilde{\varepsilon}_{h}, \varepsilon_{h}$ are listed in the tables. 
Table of errors for $h_{0}=10^{-3}, h_{1}=h_{2}=10^{-2}$

\begin{tabular}{|c|c|c|}
\hline$t^{(r)}$ & $\left(\tilde{\eta}^{(r)} ; \eta^{(r)}\right)$ & $\left(\tilde{\varepsilon}^{(r)} ; \varepsilon^{(r)}\right)$ \\
\hline 0,4 & $1 \cdot 10^{-3} ; 2 \cdot 10^{-4}$ & $1 \cdot 10^{-3} ; 1 \cdot 10^{-4}$ \\
0,5 & $2 \cdot 10^{-3} ; 3 \cdot 10^{-4}$ & $1 \cdot 10^{-3} ; 1 \cdot 10^{-4}$ \\
0,6 & $3 \cdot 10^{-3} ; 4 \cdot 10^{-4}$ & $1 \cdot 10^{-3} ; 2 \cdot 10^{-4}$ \\
0,7 & $3 \cdot 10^{-3} ; 5 \cdot 10^{-4}$ & $1 \cdot 10^{-3} ; 2 \cdot 10^{-4}$ \\
0,8 & $4 \cdot 10^{-3} ; 6 \cdot 10^{-4}$ & $2 \cdot 10^{-3} ; 2 \cdot 10^{-4}$ \\
0,9 & $5 \cdot 10^{-3} ; 7 \cdot 10^{-4}$ & $2 \cdot 10^{-3} ; 2 \cdot 10^{-4}$ \\
1,0 & $5 \cdot 10^{-3} ; 8 \cdot 10^{-4}$ & $2 \cdot 10^{-3} ; 3 \cdot 10^{-4}$ \\
\hline
\end{tabular}

Table of errors for $h_{0}=10^{-4}, h_{1}=h_{2}=5 \cdot 10^{-3}$

\begin{tabular}{|c|c|c|}
\hline$t^{(r)}$ & $\left(\tilde{\eta}^{(r)} ; \eta^{(r)}\right)$ & $\left(\tilde{\varepsilon}^{(r)} ; \varepsilon^{(r)}\right)$ \\
\hline 0,4 & $3 \cdot 10^{-3} ; 1 \cdot 10^{-4}$ & $1 \cdot 10^{-3} ; 5 \cdot 10^{-5}$ \\
0,5 & $5 \cdot 10^{-3} ; 1 \cdot 10^{-4}$ & $2 \cdot 10^{-3} ; 7 \cdot 10^{-5}$ \\
0,6 & $6 \cdot 10^{-3} ; 2 \cdot 10^{-4}$ & $2 \cdot 10^{-3} ; 9 \cdot 10^{-5}$ \\
0,7 & $8 \cdot 10^{-3} ; 2 \cdot 10^{-4}$ & $3 \cdot 10^{-3} ; 9 \cdot 10^{-5}$ \\
0,8 & $9 \cdot 10^{-3} ; 3 \cdot 10^{-4}$ & $4 \cdot 10^{-3} ; 1 \cdot 10^{-4}$ \\
0,9 & $1 \cdot 10^{-2} ; 3 \cdot 10^{-4}$ & $4 \cdot 10^{-3} ; 1 \cdot 10^{-4}$ \\
1,0 & $1 \cdot 10^{-2} ; 4 \cdot 10^{-4}$ & $5 \cdot 10^{-3} ; 1 \cdot 10^{-4}$ \\
\hline
\end{tabular}

Note that $\eta_{h}^{(r)}<\tilde{\eta}_{h}^{(r)}$ and $\varepsilon_{h}^{(r)}<\tilde{\varepsilon}_{h}^{(r)}$ for all the values of $t^{(r)}$.

We give also the following information on the errors of the methods. Write

$$
\begin{aligned}
& \tilde{\eta}_{h}=\max \left\{\tilde{\eta}_{h}^{(r)}: 1 \leq r \leq N_{0}\right\}, \\
& \eta_{h}=\max \left\{\eta_{h}^{(r)}: 1 \leq r \leq N_{0}\right\}
\end{aligned}
$$

and

$$
\tilde{\varepsilon}_{h}=\frac{1}{N_{0}} \sum_{r=1}^{N_{0}} \tilde{\varepsilon}_{h}^{(r)}, \quad \varepsilon_{h}=\frac{1}{N_{0}} \sum_{r=1}^{N_{0}} \varepsilon_{h}^{(r)} .
$$

The values of the errors $\left(\tilde{\eta}_{h}, \eta_{h}\right)$ and $\left(\tilde{\varepsilon}_{h}, \varepsilon_{h}\right)$ are listed in the following table. 


\section{Table of global errors}

\begin{tabular}{|c|c|c|}
\hline$\left(h_{0}, h_{1}=h_{2}\right)$ & $\left(\tilde{\eta}_{h} ; \eta_{h}\right)$ & $\left(\tilde{\varepsilon}_{h} ; \varepsilon_{h}\right)$ \\
\hline $10^{-3} ; 5 \cdot 10^{-2}$ & 0,$050628 ; 0,000425$ & 0,$010604 ; 0,000679$ \\
$10^{-3} ; 10^{-2}$ & 0,$005727 ; 0,000890$ & 0,$001063 ; 0,000147$ \\
$5 \cdot 10^{-4} ; 10^{-2}$ & 0,$010666 ; 0,000890$ & 0,$001979 ; 0,000147$ \\
$10^{-4} ; 10^{-2}$ & 0,$034160 ; 0,000810$ & 0,$006635 ; 0,000147$ \\
$10^{-4} ; 5 \cdot 10^{-3}$ & 0,$012884 ; 0,000447$ & 0,$002387 ; 0,000074$ \\
\hline
\end{tabular}

Note that $\eta_{h}<\tilde{\eta}_{h}$ and $\left(\varepsilon_{h}<\tilde{\varepsilon}_{h}\right)$ for all the values $h$.

Thus we see that the errors of the classical difference method are larger than the errors of the generalized Euler method. This is due to the fact that the approximation of the spatial derivatives of $z$ in the new method is better than the respective approximation of $\partial_{x} z$ in the classical case. Methods described in Theorems 2 and 3 have the potential for applications in the numerical solving of first order nonlinear differential equations.

1. Kowalski Z. A difference method for certain hyperbolic systems of nonlinear partial differential equations of first order // Ann pol. math. - 1967. - 19. - P. 313-322.

2. Kowalski Z. On the difference method for certain hyperbolic systems of nonlinear partial differential equations of the first order // Bull. Acad. pol. sci. Sér. sci. math., astron. et. phys. - 1968. - 16. - P. $297-302$.

3. Pliś A. On difference inequalities corresponding to partial differential inequalities of first order // Ann. pol. math. - 1968. - 28. - P. $179-181$.

4. Cinquini S. Sopra i sistemi iperbolici di equazioni a derivate parziali (nonlineari) in piu variabili indipendenti // Ann. mat. pura ed appl. - 1979. - 120. - P. 201-214.

5. Cinquini Cibrario M. Sopra una classe di sistemi di equazioni non lineari a derivate parziali in piu variabili indipendenti // Ibid. - 1985. - 140. - P. 223-253.

6. Tran Duc Van, Miko Tsuji, and Nguyen Duy Thai Son. The characteristic method and its generalizations for first-order nonlinear partial differential equations. - Boca Raton: Chapman \& Hall/CRC, 2000.

7. Kamont Z. Hyperbolic functional differential inequalities and applications. - Dordrecht: Kluwer Acad. Publ., 1999.

8. Baranowska A., Kamont Z. Finite difference approximations for nonlinear first order partial differential equations // Univ. Iagell. Acta Math. - 2002. - 40. - P. 15-30.

9. Godlewski E., Raviart P. A. Numerical approximation of hyperbolic systems of conservation laws. - New York: Springer, 1996.

10. Magomedov K. M., Kholodov A. S. Mesh-characteristics numerical methods. - Moscow: Nauka, 1988 (in Russian).

11. Szarski J. Differential inequalities. - Warszawa: Polish Sci. Publ., 1967.

12. Lakshmikantham V., Leela S. Differential and integral inequalities. - New York; London: Acad. Press, 1969.

Received 11.06.2003 